\title{
Características clínicas e Esquema de Tratamento Medicamentoso de Infecçóes por Staphylococcus aureus: uma possível proposta de identificaçáo para o laboratorista e tratamento para o clínico
}

\section{Clinical aspects and medicinal treatment scheme for Staphylococcus aureus infections: a possible proposal of identification for the laboratory technician and treatment for the clinician}

\author{
Thaina Miranda da Costa ${ }^{1}$ \\ Marcos Gabriel Pinheiro ${ }^{1}$ \\ Claudete Araújo Cardoso ${ }^{2}$ \\ Marcelo Paraiso Alves ${ }^{4}$ \\ Fábio Aguiar-Alves ${ }^{1,3}$
}

\begin{abstract}
Resumo
A área de ensino, no decorrer de sua trajetória histórica, tem se deparado com problemas de diversas ordens, como por exemplo: a formação de professores e em decorrência na formaçáo dos profissionais da área da saúde. Ao considerar que a formação docente ainda é caracterizada por um viés conteudista, distante da experimentação, acarreta consequências no campo do ensino, pois os mesmos discentes que outrora obtiveram lacunas em sua formaçấo, na atualidade militam no campo da docência em múltiplos campos do saber reproduzindo tais práticas descontextualizadas. Para entender a prática docente e como modificá-la, alguns estudos consideram a importância da perspectiva do professor investigador e as necessidades formativas indispensáveis para a melhoria do ensino de Ciências. Em função da abrangência destes aspectos, há necessidade de se aliar os conhecimentos específicos de variados campos do saber, os aspectos metodológicos diversificados e os princípios históricos e epistemológicos da construção do conhecimento científico. Portanto, sabendo das lacunas que envolvem o processo de formaçáo do laboratorista esse artigo tem como objetivo discutir uma proposta de identificaçáo clínica para laboratorista, mais especificamente para o Staphylococcus aureus, na intenção de servir como um material didático para minizar tais impactos no processo de formação de tais profissionais. O Staphylococcus aureus é bem conhecido pela sua capacidade para adquirir genes de resistência a drogas antimicrobianas. Este patógeno é historicamente associado a infecçôes relacionadas à assistência à saúde e pode se disseminar rapidamente entre os indivíduos saudáveis. Surtos de infecçôes causadas por cepas de Staphylococcus aureus resistente a meticilina associados a comunidade (CA-MRSA) têm sido relatados em todo o mundo, incluindo o Brasil, onde existe atualmente uma epidemia de CA-MRSA. Pensando nesta problemática, apresentamos aqui, uma revisão sobre a utilização adequada das metodologias fenotípicas e genotípicas de caracterização dos isolados de Staphylococcus aureus de grande importância no ambiente hospitalar, assim como a caracterizaçáo clínica das doenças estafilocócicas e o tratamento medicamentoso adequado para combater estas infecçôes de maneira rápida e de acordo com as modificaçôes por aquisição, por este organismo, de mecanismos de resistência.
\end{abstract}

Palavras-chave: METHICILLIN-RESISTANT Staphylococcus aureus, colonizaçâo nasal, infecção, genótipos.

\footnotetext{
${ }^{1}$ Programa de Pós-Graduação em Patologia - Faculdade de Medicina - Universidade Federal Fluminense - Niterói - Brasil

${ }^{2}$ Programa de Pós-Graduação em Ciências Médicas - Faculdade de Medicina - Universidade Federal Fluminense - Niterói - Brasil

${ }^{3}$ Polo Universitário de Nova Friburgo - Universidade Federal Fluminense - Nova Friburgo - Brasil

${ }^{4}$ Mestrado Profissional em Ensino em Ciências e Meio Ambiente - Centro Universitário de Volta Redonda - UniFOA - Volta Redonda - Rio de Janeiro
} 


\begin{abstract}
The area of education, in the course of its historical trajectory, has been facing problems of various orders, such as: teacher education and as a result of the training of health professionals. When considering that teacher training is still characterized by a bias content way, away from experimentation, has consequences in the field of education, as these students who once had gaps in their education, today activists in the field of teaching in many fields of knowledge playing such practices out of context. To understand how the teaching practice and modify it, some studies consider the importance of the perspective of the researcher and teacher training needs necessary for the improvement of science teaching. Depending on the scope of these aspects, it is necessary to combine the expertise of various fields of knowledge, methodological aspects and the diverse historical and epistemological principles of construction of scientific knowledge. Therefore, knowing the gaps involving the formation process of the laboratory technician this article aims to discuss a proposal for identifying clinical laboratory technician, specifically for Staphylococcus aureus, intending to serve as an educational material for minizar such impacts in the training process of such professionals. Staphylococcus aureus is well known for its ability to acquire resistance genes antimicrobial drugs. This pathogen is historically associated with infections related to health care and can spread rapidly among healthy individuals. Outbreaks of infections caused by strains of methicillin-resistant Staphylococcus aureus associated with community (CA-MRSA) have been reported worldwide, including Brazil, where there is currently an epidemic of CA-MRSA. Thinking about this issue, we present here a review of the use of appropriate methodologies phenotypic and genotypic characterization of Staphylococcus aureus of great importance in the hospital environment, as well as the clinical characterization of staphylococcal diseases and appropriate medical treatment to combat these infections so and rapid changes in accordance with the acquisition, by this organism of resistance mechanisms.
\end{abstract}

\section{INTRODUÇÃO}

O Ensino das Ciências tem se deparado, no decorrer de sua trajetória histórica, com problemas tanto em seus aspectos teórico-metodológicos como na formação de professores e em decorrência na formação dos profissionais da área da saúde, pois ao considerar que a formação dos docentes, historicamente, perpassou por um viés conteudista, mecânico e linear, distante da experimentação, esses mesmos discentes de outrora, militam hoje no campo da docência em múltiplos campos do saber reproduzindo tais práticas pedagógicas. Para entender a prática docente e como modificála, alguns estudos consideram a importância da perspectiva do professor investigador e as necessidades formativas indispensáveis para a melhoria do ensino de Ciências. Em função da abrangência destes aspectos, há necessidade de se aliar os conhecimentos específicos de variados campos do saber, os aspectos metodológicos diversificados e os princípios históricos e epistemológicos da construção do conhecimento científico (42, 43, 44 e 45). Assim, ciente das lacunas que envolvem o processo de formação dos profissionais (mecânica, memorização, distante de experimentaçôes) da saúde, em especial, dos laboratoristas, esse artigo tem como objetivo discutir uma proposta didática na intenção de auxiliar o processo formação do laboratorista, mais especificamente para a identificação do Staphylococcus aureus.
A tentativa da referida proposta é criar um material didático para minizar os impactos provocado pela influência da Pedagogia Tecnicista presente no processo de formação de tais profissionais. Seguindo essa mesma perspectiva, Mitre et. al. (2008) (46) menciona que: "historicamente, a formação dos profissionais de saúde tem sido pautada no uso de metodologias conservadoras (ou tradicionais), sob forte influência do mecanicismo de inspiração cartesiananewtoniana, fragmentado e reducionista" (46).

Para o autor, o pensamento moderno influenciou a dicotomia que se configura no processo de ensino burocratizando o sistema educacional, manifestando-se também por meio das subdivisóes da universidade em centros e departamentos e dos cursos em períodos ou séries e em disciplinas estanques.

Dessa forma, o processo de ensino-aprendizagem, influenciado pelo paradigma supracitado, desenvolve suas ações restritas à reprodução do conhecimento, no qual o professor se configura apenas como um mero técnico transmissor de conteúdos, cabendo ao discente, o papel de receptor, uma atitude passiva e reprodutora de conceitos, nomes, datas, dentre outros. Pensando na possibilidade de minimizar a lacuna que emerge da fragmentação entre teoria e prática no processo de ensino-aprendizagem, o artigo se estruturou a partir da seguinte configuração: No primeiro momento, apresentamos as características principais do Staphylococcus aureus; na segunda parte do texto procuramos 
discutir o processo de Identificação Fenotípica de Staphylococcus aureus; na terceira parte a Identificação Genotípica; na quarta parte as Características Epidemiológicas e Clínicas; E por fim, o tratamento medicamentoso.

\section{DISSEMINAÇÃO E CARACTERÍSTICAS MOLE- CULARES DE STAPHYLOCOCCUS AUREUS}

$S$. aureus pode ser intermitente ou permanentemente presente na microbiota de narinas, pele, garganta e trato intestinal de alguns indivíduos. Esta colonização permite que esses sujeitos atuem como disseminadores deste microorganismo. Além disso, esses indivíduos estão em maior risco de infecção $(5,7,30)$. Atualmente, acredita-se que 30\% dos seres humanos têm colonização nasal assintomática por S.aureus $(5,33)$.

A transmissão ocorre por contato direto, geralmente através da pele de uma pessoa colonizada ou infectada com lesôes abertas. Outras rotas com fômites e mãos dos profissionais de saúde também são importantes meios de transmissão. Mais recentemente, vários surtos de Staphylococcus aureus resistentes a meticilina (MRSA) têm sido associados com a presença de amostras em alimentos de origem animal (31).

A propagação é facilitada por várias razóes, incluindo a migração da população, profissionais de saúde especialmente, combinada com métodos ineficazes para controlar a transmissão de MRSA de pacientes colonizados e infectados, juntamente com o uso inadequado de antimicrobianos $(6,7,8,9,10,20,21,22,30,33)$.

As infecçóes causadas por MRSA têm atingido uma cota global e estão aumentando em hospitais e na comunidade, inclusive nos países que anteriormente tinham baixa prevalência de MRSA, expondo uma diversidade significativa de clones identificados.

Resistência a meticilina é conferida pelo gene mec A que é transportado por um elemento genético móvel, conhecido como Cassete Cromossômico Estafilocócico mec (SCCmec) que codifica uma proteína de ligação à penicilina modificada, chamada PBP2a ou PBP2', que possui uma baixa afinidade para beta-lactâmicos, incluindo penicilinas, cefalosporinas e carbapenêmicos $(9,18,19,37,41)$.

De acordo com publicações, até agora, há 11 alótipos diferentes de SCCmec, os tipos I a XI, que foram revelados entre as cepas de MRSA $(5,9,18,19,37,41)$. O SCCmec apresenta componentes genéticos do complexo gene MEC e o gene responsável pela recombinação do cassete cromossômico (CCR) (5,9,10,17,19,37,41). Variaçóes dentro desses complexos gênicos servem como base primária para classificação dos vários tipos de SCCmec $(5,19,37,38,41)$.
PVL (Leucocidina Panton-Valentine)

A importância da toxina PVL na virulência e epidemiologia de CA-MRSA é um fato de grande controvérsia $(23,24,29)$. A PVL é uma toxina secretada por S.aureus, composta por duas subunidades chamadas LukS-PV e LukF-PV. Ela está relacionada com a formação de poros na membrana de leucócitos polimorfonucleares de acolhimento e macrófagos $(18,23)$. A PVL pode ser produzida por estirpes sensíveis a beta lactâmicos e por algumas estirpes de CA-MRSA (8).

A diversidade de estudos sobre a importância da toxina PVL indica que talvez isso possa ser interpretado como uma exacerbação e não como a causa da patogênese comum provacada por CA-MRSA, como pneumonia e ostemielite (29).

As técnicas moleculares para genotipagem de isolados de S.aureus permitem um estudo mais aprofundado deste patógeno, permitindo uma compreensão mais apurada da propagação e da evolução. A técnica de MLST (Multilocus Sequence Typing), por exemplo, permite que os clones sejam classificados em tipos, de acordo com a sequência (ST). Por sua vez, os STs são agrupados em complexos clonais (CC) $(15,32,36,37,38,40)$.

No Brasil, o MRSA é uma das principais causas de infecçóes relacionadas à assistência à saúde. $\mathrm{O}$ clone endêmico de MRSA, na maioria dos hospitais de norte a sul, desde 1994, se caracteriza por ser multirresistente, sendo capaz de produzir biofilme e, na maioria dos casos não produz toxina PVL $(2,3,11,33)$. Este clone endêmico brasileiro (BEC) é classificado como SCCmec IIIA-ST239 (CC8) e pertence ao mesmo complexo clonal de MRSA identificado pela primeira vez no mundo $(11,24,27,28,34,35,36)$. Apesar disso, estudos relataram um aumento da presença de não multirresistentes MRSA SCCmec tipo IV, característica de amostras associadas a comunidades em indivíduos internados em hospitais, mostrando uma mudança no perfil molecular de amostras em hospitais brasileiros.

MRSA é um dos patógenos causadores de endocardite infecciosa no Brasil (13). O primeiro relato de doença causada por CA-MRSA no país ocorreu em 2008, por uma amostra de SCCmec tipo IV e PVL positivo (12).

\section{IDENTIFICAÇÁO FENOTÍPICA DE STAPHYLO- COCCUS AUREUS}

A coleta pode ser realizada através de rotação de swab, seco e estéril, no vestíbulo anterior das duas narinas de cada paciente. Os swabs coletados são semeados em ágar sangue ou caldo BHI (infusão de cérebro e coração), o qual é incubado em estufa a $37^{\circ} \mathrm{C}$ por 24 horas. Havendo crescimento, 
as colônias são semeadas em ágar manitol salgado (BBL Mannitol Salt Agar - Becton, Dickinson and Company).

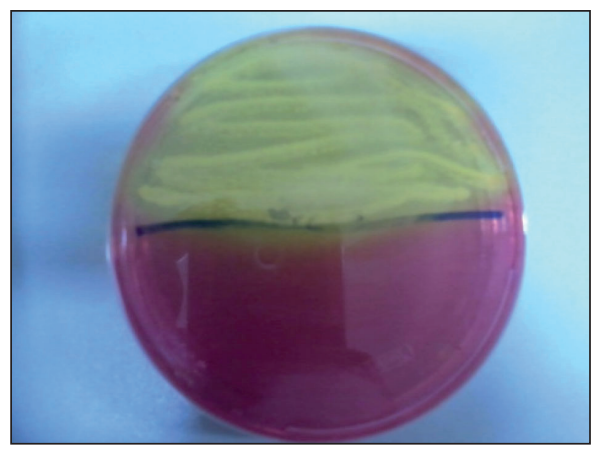

Figura 1. Placa de Manitol

Típicas colônias de Staphylococcus aureus são submetidas ao teste de Gram, ao teste de catalase e também ao teste de coagulase, para confirmação desta bactéria (15). O método de difusão em disco é utilizado para observar a resistência à meticilina com o uso da temperatura e do antibiótico correto. A resistência é detectada por difusão em disco de oxacilina com temperatura à $35^{\circ} \mathrm{C}$ em ágar Mueller Hinton. Os frascos contendo os discos de papel, para o teste de sensibilidade, são armazenados resfriados a temperatura de $8^{\circ} \mathrm{C}$ ou menos e retirados da geladeira uma hora antes da realização da técnica, a fim de minimizar a condensação quando o ar quente entrar em contato com disco. O inóculo bacteriano será ajustado em 0.5 de McFarland seguindo as orientaçôes da norma global consensual do CLSI (14).

\section{IDENTIFICAÇÁO GENOTÍPICA}

O isolamento do DNA bacteriano é realizado com Kit QIAamp ${ }^{\oplus}$ DNA mini (QUIAGEN) seguindo protocolo do produto (25).
PCR (reaçáo em cadeia da polimerase) baseia-se na amplificação da região específica do DNA. Os produtos de amplificação são submetidos à eletroforese em gel de agarose, utilizando-se padrão de tamanho molecular de 100 pb e corados em solução contendo brometo de etídio, visualizados e foto-documentados sob luz Ultra Violeta (UV) $(9,39)$.

Após o teste molecular para a confirmação de resistência à meticilina, todas as amostras de MRSA são submetidas à Multiplex-PCR e a duas reaçóes uniplex-PCR, para tipagem e subtipagem do cassete SCCmec.

Os genes que codificam a PVL (lukS-PV e lukF-PV) são co-amplificados em uma reação única através de reaçóes de PCR como descrito por Lina et al (1999).

As amostras também são submetidas a Eletroforese em Gel de Campo Pulsado (PFGE), para caracterização do perfil do clone circulante. A determinação do tipo estrutural do elemento mec é realizada por um sistema de multiplexPCR, como descrito por Oliveira e Lencastre (2002), e por reaçóes de PCR adicionais para determinação de variantes do SCCmec IV, os subtipos IVa e IVb (26).

A combinação das bandas amplificadas pelo esquema multiplex definem o tipo de SCCmec, conforme descrito por Oliveira \& Lencastre (2002).

A figura 2 mostra a PCR para o gene de PVL e os genes mecA. As amostras foram classificadas como “+” para caracterizar a presença dos genes e “_" para amostras que são negativas para estes genes. A imagem mostra uma coloração de brometo de etídio de um gel de agarose a 1,5\% contendo as seguintes amostras: $\mathrm{M}$ - marcador do tamanho molecular (100 pb) e amostras PVL+: 1-7; 8-C-(HU25); 9-C+ (WB45); 10 Branco; M- marcador do tamanho molecular (100 bp); Amostras do Meca+: 11-17; 18-C-(MSSA), 19C + (WB 45), 20-Branco.

\section{Figura 2}

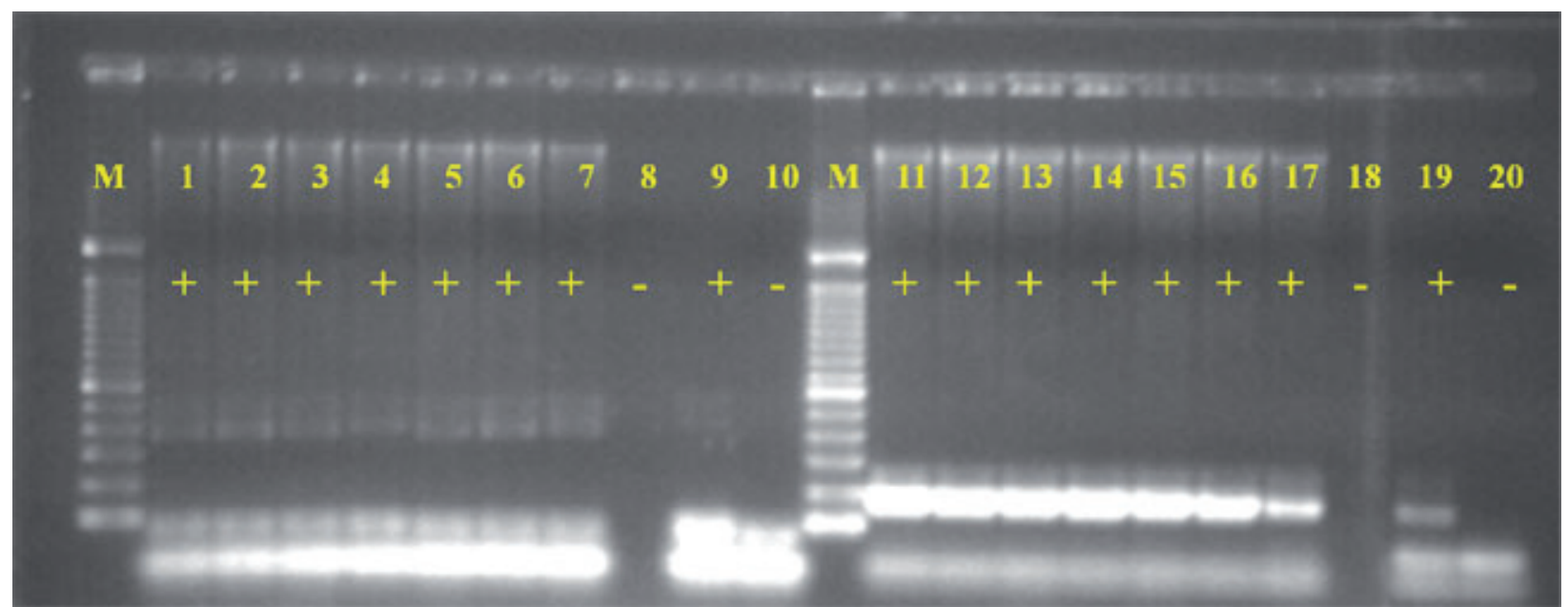




\section{CARACTERÍSTICAS EPIDEMIOLÓGICAS E CLÍNICAS}

$\mathrm{Na}$ América Latina, estudos pontuais indicam que MRSA é a principal causa de infecçóes relacionadas a assistência a saúde, e vem crescendo a prevalência desse microrganismo nas infecções adquiridas na comunidade (16). A epidemiologia dos MRSA está em constante mudança e o estudo dos clones circulantes e do perfil de resistência antimicrobiana pode variar consideravelmente conforme a regiāo. A vigilância epidemilógica local é fundamental para dar suporte na escolha do tratamento empírico de antibióticos, escolha esta baseada no conhecimento dos patógenos circulantes.

Em decorrência da fácil transmissão e adaptação deste patógeno, em adição aos inevitáveis fatores de risco, a erradicação total das infecçóes relacionadas à assistência à saúde é dificultada (24)

A maioria das manifestaçóes clínicas relacionadas com CA-MRSA são infecçôes de pele e subcutâneo. No entanto, algumas amostras parecem ser particularmente virulentas, sendo causadoras de infecçóes de tecido degenerativas como fasciite necrosante, que pode evoluir para forma fulminante, sepse grave e pneumonia necrosante, condiçóes estas que raramente eram vistas antes da emergência de cepas de CA-MRSA $(4,5)$.

As infecçóes estafilocócicas variam de localizadas, geralmente superficiais, até profundas, com elevada gravidade (8).

As infecçôes superficiais, como por exemplo, acnes, furúnculos e infecçóes de feridas, em geral decorrem da invasão dos tecidos cutâneo e/ou subcutâneo por bactérias presentes na pele ou mucosas. Já as profundas, salvo algumas exceçóes, são freqüentemente decorrentes de bacteremias originadas nos focos de infecçôes superficiais, como é o caso de sepse, com elevada mortalidade (21). A pneumonia é um quadro de infecção profunda que está geralmente associado ao uso de ventilação mecânica (1).

Há ainda quadros tóxicos, como a síndrome do choque tóxico, a doença de Ritter (síndrome da pele escaldada), o impetigo bolhoso e a intoxicação alimentar (2).

Em decorrência da fácil transmissão e adaptação deste patógeno, em adição aos inevitáveis fatores de risco, a erradicação total das infecções relacionadas à assistência à saúde é dificultada (12).

\section{TRATAMENTO MEDICAMENTOSO}

As infecções causadas por cepas resistentes geralmente ocorrem em ondas epidêmicas que são iniciadas por um ou poucos clones bem sucedidos. A resistência do Staphylococcus aureus aos antibióticos pode ser visualizada em vários estágios, porém há quatro principais: o primeiro está entre as décadas de 40 a 60, o segundo entre os anos 60 a 80, já o terceiro estágio ocorreu na década de 80 e o quarto vai desta última até os tempos atuais, conforme a figura 3 (5).

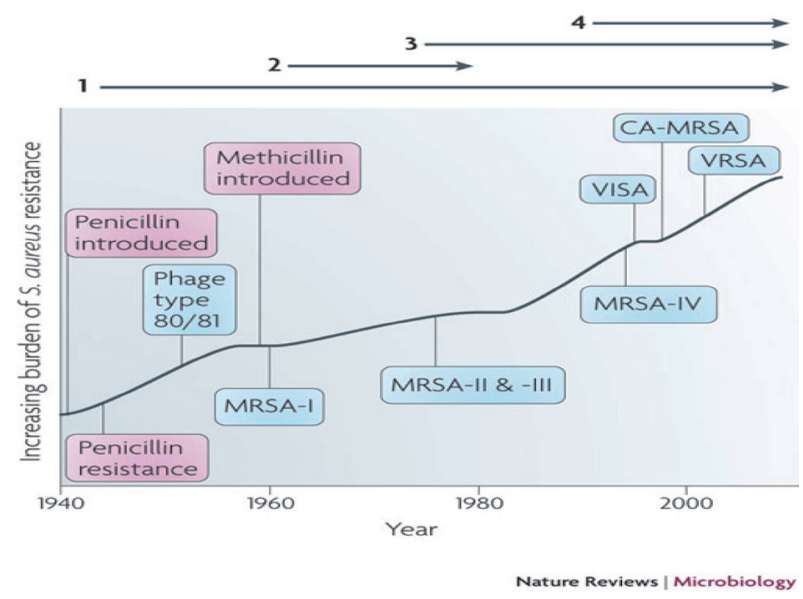

Figura 3 - As principais etapas de resistência aos antimicrobianos. Fonte: Chambers \& Deleo; Waves of resistance: Staphylococcus aureus in the antibiotic era; 2009)

Em 1942, dois anos após a introdução da penicilina na prática clínica, a resistência a droga já havia sido detectada em hospitais e no início da década de 50 já era um problema notável em comunidades numa escala global (9).

Para o tratamento do $S$. aureus sensível à meticilina, a terapia de escolha para os pacientes que se encontram clinicamente estáveis é uma cefalosporina de primeira geração (cefalexina ou cefadroxil). Para os indivíduos que estão instáveis clinicamente, com indicação de terapia antimicrobiana intravenosa, indica-se a oxacilina. Neste pacientes com terapia inicial intravenosa que evoluem com melhora clínica progressiva, pode-se prescrever a terapia seqüencial com cefalosporina de primeira geraçáo a fim de se completar o tratamento. A duração da terapia dependerá do foco infeccioso, devendo ser realizada por um período mínimo de 10 dias nas infecçóes de pele (usualmente se utiliza de 10 a 14 dias).

No tratamento do CA-MRSA em pacientes clinicamente estáveis, as opções terapêuticas são o sulfametoxazoltrimetoprim e a clindamicina. Nos indivíduos com quadro clínico grave, a terapia antimicrobiana de escolha é a vancomicina (22).

$\mathrm{Na}$ abordagem terapêutica das infecçóes relacionadas a MRSA de origem hospitalar, o fármaco de escolha é a vancomicina. Uma outra opção de terapia é a linezolida, indicada para os casos de intolerância e/ou resistência à vancomicina (22)

\section{CONSIDERAÇÓES FINAIS}

O uso indiscriminado de antibióticos é um fator importante que contribui para o surgimento de novas cepas 
resistentes de $S$. aureus. A correta identificação de MRSA é essencial para garantir o tratamento adequado dos pacientes. Além disso, existe uma necessidade para manter a vigilância e permitir a identificação de alteraçôes no perfil de resistência de clones a partir de uma região, permitindo a atualização do conhecimento da epidemiologia de MRSA e medidas mais apropriadamente orientadas para controlar a sua difusão.

\section{REFERENCES}

10. Allegranzi, B. et al. Burden of endemic health-care-associated infection in developing countries: systematic review and meta-analysis. Lancet, 377(9761), 228241, 2011.

11. Balaban, N. et al. Autoinducer of virulence as a target for vaccine and therapy against Staphylococcus aureus. Science, 280(5362), 438-440, 1998.

12. Berglund, C.; Molling, P.; Sjoberg, L. and Soderquist, B. Predominance of staphylococcal cassette chromosome mec (SCCmec) type IV among methicillin-resistant Staphylococcus aureus (MRSA) in a Swedish county and presence of unknown SCCmec types with Panton-Valentine leukocidin genes. (2005) Clin Microbiol Infect. 11(6), 447-456.

13. Boyle-Vavra S, Daum RS. Community-acquired methicillin-resistant Staphylococcus aureus: the role of Panton-Valentine leukocidin. Lab Invest 2007; 87:3-

14. Chambers \& Deleo; Waves of resistance: Staphylococcus aureus in the antibiotic era; (2009)

15. Chambers, H.F. and Deleo, F.R. Waves of resistance: Staphylococcus aureus in the antibiotic era. (2009) Nat Rev Microbiol. 7(9), 629-641.

16. Conly, J.M. and Johnston, B.L. The emergence of methicillin-resistant Staphylococcus aureus as a community-acquired pathogen in Canada. (2003) Can J Infect Dis. 14(5), 249-251.

17. Dancer, S.J. The effect of antibiotics on methicillinresistant Staphylococcus aureus. (2008) J Antimicrob Chemother. 61(2), 246-253.
18. Deurenberg, R.H. and Stobberingh, E.E. The evolution of Staphylococcus aureus. (2008) Infect Genet Evol. 8(6), 747-763.

19. Deurenberg, R.H. and Stobberingh, E.E. The molecular evolution of hospital- and community-associated methicillin-resistant Staphylococcus aureus. (2009) Curr Mol Med. 9(2), 100-115.

20. dos Santos Soares, M.J.; da Silva-Carvalho, M.C.; Ferreira-Carvalho, B.T. and Figueiredo, A.M. Spread of methicillin-resistant Staphylococcus aureus belonging to the Brazilian epidemic clone in a general hospital and emergence of heterogenous resistance to glycopeptide antibiotics among these isolates. (2000) J Hosp Infect. 44(4), 301-308.

21. Fortes, C.Q.; Espanha, C.A.; Bustorff, F.P.; Zappa, B.C.; Ferreira, A.L.; Moreira, R.B.; Pereira, N.G.; Fowler, V.G., Jr. and Deshmukh, H. First reported case of infective endocarditis caused by community-acquired methicillin-resistant Staphylococcus aureus not associated with healthcare contact in Brazil. (2008) Braz J Infect Dis. 12(6), 541-543.

22. Fowler, V.G., Jr.; Miro, J.M.; Hoen, B.; Cabell, C.H.; Abrutyn, E.; Rubinstein, E.; Corey, G.R.; Spelman, D.; Bradley, S.F.; Barsic, B.; Pappas, P.A.; Anstrom, K.J.; Wray, D.; Fortes, C.Q.; Anguera, I.; Athan, E.; Jones, P.; van der Meer, J.T.; Elliott, T.S.; Levine, D.P. and Bayer, A.S. Staphylococcus aureus endocarditis: a consequence of medical progress. (2005) JAMA. 293(24), 3012-3021.

23. Franklin R. Cockerill; Mathew A. Wikler; Karen Bush; Michael N. Dudley; George M. Eliopoulos; Dwight J. Hardy; David W. Hecht; Janet A. Hindler; Jean B. Patel; Mair Powel; Richard B. Thomson Jr.; John D. Turnide; Melvin P. Weinstein; Barbara L. Zilmmer; Mary Jane Ferraro; Jana M. Zwenson. Performance Standards for Antimicrobial Susceptibility Testing; Twenty-First Informational Supplement. (2011) Clinical and Laboratory Standards Institute.

24. Grothe, C.; Belasco A.G.S.; Bittencourt, A.R.C.; Vianna L.A.C.; Sesso, R.C.C.; Barbosa, D.A. Incidence of Bloodstream Infection Among Patients on Hemodialysis by Centrous Venous Catheter. Rev. Latino-Am. Enfermagem Jan-Feb 2010; 18(1): 73-80. 
25. Guzmán-Blanco M, Mejía C, Isturiz R et al. Epidemiology of methicillin-resistant Staphylococcus aureus (MRSA) in Latin America. Int J Antimicrob Agents. 2009; 34 (4): 304-308.

26. Ito, T.; Ma, X.X.; Takeuchi, F.; Okuma, K.; Yuzawa, H. and Hiramatsu, K. Novel type V staphylococcal cassette chromosome mec driven by a novel cassette chromosome recombinase, ccrC. (2004) Antimicrob Agents Chemother. 48(7), 2637-2651.

27. Katayama, Y.; Ito, T. and Hiramatsu, K. A new class of genetic element, staphylococcus cassette chromosome mec, encodes methicillin resistance in Staphylococcus aureus. (2000) Antimicrob Agents Chemother. 44(6), 1549-1555.

28. Kondo, Y.; Ito, T.; Ma, X.X.; Watanabe, S.; Kreiswirth, B.N.; Etienne, J. and Hiramatsu, K. Combination of multiplex PCRs for staphylococcal cassette chromosome mec type assignment: rapid identification system for mec, ccr, and major differences in junkyard regions. (2007) Antimicrob Agents Chemother. 51(1), 264-274.

29. Lina G, et al. Involvement of Panton-Valentine leukocidinproducing Staphylococcus aureus in primary skin infections and pneumonia. Clin Infect Dis, 29: 1128 - 1132, 1999.

30. Lindsay, J. A.; Holden, M. T. Staphylococcus aureus: superbug, super genome? Trends Microbiol, 12(8), 378-385, 2004.

31. Liu C, Bayer A, Cosgrove SE, Daum RS, Fridkin SK, Gorwitz RJ, et al. Clinical Practice Guidelines by the Infectious Diseases Society of America for the Treatment of Methicilin-Resistant Staphylococcus aureus infections in adults and children. Clinical Infectious Diseases 2011; 52: 1-38.

32. Lo, W.T. and Wang, C.C. Panton-Valentine leukocidin in the pathogenesis of community-associated methicillin-resistant Staphylococcus aureus infection. (2011) Pediatr Neonatol. 52(2), 59-65.

33. Lu, P. L. et al. Risk factors and molecular analysis of community methicillin-resistant Staphylococcus aureus carriage. J Clin Microbiol, 43(1), 132-139, 2005.

34. N.Gardella; S.Murzicato; S. Di Gregorio; A. Cuirolo; J.Desse; F. Crudo; G.Gutking; M. Mollerach. Prevalence and Characterization of methicilin- resistent Sthaphylococcus aureus among healthy children in city of Argentina. Infection, Genetics and Evolution 11 (2011) 1066-1071
35. Okuma. K. et al. Dissemination of new methicillinresistant Staphylococcus aureus clones in the community. J Clin Microbiol, 40: 4289 - 4294, 2002.

36. Oliveira, D.C.; Tomasz, A. and de Lencastre, H. The evolution of pandemic clones of methicillin-resistant Staphylococcus aureus: identification of two ancestral genetic backgrounds and the associated mec elements. (2001) Microb Drug Resist. 7(4), 349-361.

37. Oliveira, G.A.; Faria, J.B.; Levy, C.E. and Mamizuka, E.M. Characterization of the Brazilian endemic clone of methicillin-resistant Staphylococcus aureus (MRSA) from hospitals throughout Brazil. (2001) Braz J Infect Dis. 5(4), 163-170.

38. Otto, M. A MRSA-terious enemy among us: end of the PVL controversy? (2011) Nat Med. 17(2), 169-170.

39. Ramos, R.L.; Teixeira, L.A.; Ormonde, L.R.; Siqueira, P.L.; Santos, M.S.; Marangoni, D. and Figueiredo, A.M. Emergence of mupirocin resistance in multiresistant Staphylococcus aureus clinical isolates belonging to Brazilian epidemic clone III::B:A. (1999) J Med Microbiol. 48(3), 303-307.

40. Rizek, C.F.; Matte, M.H.; Dropa, M.; Mamizuka, E.M.; de Almeida, L.M.; Lincopan, N.; Matte, G.R. and Germano, P.M. Identification of Staphylococcus aureus carrying the mecA gene in ready-to-eat food products sold in Brazil. (2011) Foodborne Pathog Dis. 8(4), 561-563.

41. Robinson, D.A. and Enright, M.C. Multilocus sequence typing and the evolution of methicillin-resistant Staphylococcus aureus. (2004) Clin Microbiol Infect. 10(2), 92-97.

42. Rodriguez-Noriega, E.; Seas, C.; Guzman-Blanco, M.; Mejia, C.; Alvarez, C.; Bavestrello, L.; Zurita, J.; Labarca, J.; Luna, C.M.; Salles, M.J. and Gotuzzo, E. Evolution of methicillin-resistant Staphylococcus aureus clones in Latin America. (2010) Int J Infect Dis. 14(7), e560-566.

43. ALLEGRANZI B. et al. Burden of endemic health-care-associated infection in developing countries: systematic review and meta-analysis. Lancet, 377(9761), 228-241, 2011.

44. BALABAN, N. et al. Autoinducer of virulence as a target for vaccine and therapy against Staphylococcus aureus. Science, 280(5362), 438-440, 1998. 
45. BERGLUND, C.; Molling, P.; Sjoberg, L. and Soderquist, B. Predominance of staphylococcal cassette chromosome mec (SCCmec) type IV among methicillin-resistant Staphylococcus aureus (MRSA) in a Swedish county and presence of unknown SCCmec types with Panton-Valentine leukocidin genes. (2005) Clin Microbiol Infect. 11(6), 447-456.

46. BOYLE-VAVRA S, Daum RS. Community-acquired methicillin-resistant Staphylococcus aureus: the role of Panton-Valentine leukocidin. Lab Invest 2007; 87:3-

47. CHAMBERS \& Deleo; Waves of resistance: Staphylococcus aureus in the antibiotic era; (2009)

48. CHAMBERS, H.F. and Deleo, F.R. Waves of resistance: Staphylococcus aureus in the antibiotic era. (2009) Nat Rev Microbiol. 7(9), 629-641.

49. CONLY, J.M. and Johnston, B.L. The emergence of methicillin-resistant Staphylococcus aureus as a community-acquired pathogen in Canada. (2003) Can J Infect Dis. 14(5), 249-251.

50. DANCER, S.J. The effect of antibiotics on methicillin-resistant Staphylococcus aureus. (2008) J Antimicrob Chemother. 61(2), 246-253.

51. DeURENBERG, R.H. and Stobberingh, E.E. The evolution of Staphylococcus aureus. (2008) Infect Genet Evol. 8(6), 747-763.

52. DEURENBERG, R.H. and Stobberingh, E.E. The molecular evolution of hospital- and community-associated methicillin-resistant Staphylococcus aureus. (2009) Curr Mol Med. 9(2), 100-115.

53. DOS SANTOS SOARES, M.J.; da Silva-Carvalho, M.C.; Ferreira-Carvalho, B.T. and Figueiredo, A.M. Spread of methicillin-resistant Staphylococcus aureus belonging to the Brazilian epidemic clone in a general hospital and emergence of heterogenous resistance to glycopeptide antibiotics among these isolates. (2000) J Hosp Infect. 44(4), 301-308.

54. FORTES, C.Q.; Espanha, C.A.; Bustorff, F.P.; Zappa, B.C.; Ferreira, A.L.; Moreira, R.B.; Pereira, N.G.; Fowler, V.G., Jr. and Deshmukh, H. First reported case of infective endocarditis caused by communityacquired methicillin-resistant Staphylococcus aureus not associated with healthcare contact in Brazil. (2008) Braz J Infect Dis. 12(6), 541-543.
55. FOWLER, V.G., Jr.; Miro, J.M.; Hoen, B.; Cabell, C.H.; Abrutyn, E.; Rubinstein, E.; Corey, G.R.; Spelman, D.; Bradley, S.F.; Barsic, B.; Pappas, P.A.; Anstrom, K.J.; Wray, D.; Fortes, C.Q.; Anguera, I.; Athan, E.; Jones, P.; van der Meer, J.T.; Elliott, T.S.; Levine, D.P. and Bayer, A.S. Staphylococcus aureus endocarditis: a consequence of medical progress. (2005) JAMA. 293(24), 3012-3021.

56. FRANKLIN R. Cockerill; Mathew A. Wikler; Karen Bush; Michael N. Dudley; George M. Eliopoulos; Dwight J. Hardy; David W. Hecht; Janet A. Hindler; Jean B. Patel; Mair Powel; Richard B. Thomson Jr.; John D. Turnide; Melvin P. Weinstein; Barbara L. Zilmmer; Mary Jane Ferraro; Jana M. Zwenson. Performance Standards for Antimicrobial Susceptibility Testing; Twenty-First Informational Supplement. (2011) Clinical and Laboratory Standards Institute.

57. GROTHE, C.; Belasco A.G.S.; Bittencourt, A.R.C.; Vianna L.A.C.; Sesso, R.C.C.; Barbosa, D.A. Incidence of Bloodstream Infection Among Patients on Hemodialysis by Centrous Venous Catheter. Rev. Latino-Am. Enfermagem Jan-Feb 2010; 18(1): 73-80.

58. GUZMÁN-BLANCO M, Mejía C, Isturiz R et al. Epidemiology of methicillin-resistant Staphylococcus aureus (MRSA) in Latin America. Int J Antimicrob Agents. 2009; 34 (4): 304-308

59. ITO, T.; Ma, X.X.; Takeuchi, F.; Okuma, K.; Yuzawa, H. and Hiramatsu, K. Novel type V staphylococcal cassette chromosome mec driven by a novel cassette chromosome recombinase, ccrC. (2004) Antimicrob Agents Chemother. 48(7), 2637-2651.

60. KATAYAMA, Y.; Ito, T. and Hiramatsu, K. A new class of genetic element, staphylococcus cassette chromosome mec, encodes methicillin resistance in Staphylococcus aureus. (2000) Antimicrob Agents Chemother. 44(6), 1549-1555.

61. KONDO, Y.; Ito, T.; Ma, X.X.; Watanabe, S.; Kreiswirth, B.N.; Etienne, J. and Hiramatsu, K. Combination of multiplex PCRs for staphylococcal cassette chromosome mec type assignment: rapid identification system for mec, ccr, and major differences in junkyard regions. (2007) Antimicrob Agents Chemother. 51(1), 264-274.

62. LINA G, et al. Involvement of Panton-Valentine leukocidin-producing Staphylococcus aureus in primary skin infections and pneumonia. Clin Infect Dis, 29: $1128-1132,1999$. 
63. LINDSAY, J. A.; Holden, M. T. Staphylococcus aureus: superbug, super genome? Trends Microbiol, 12(8), 378-385, 2004.

64. LIU C, Bayer A, Cosgrove SE, Daum RS, Fridkin SK, Gorwitz RJ, et al. Clinical Practice Guidelines by the Infectious Diseases Society of America for the Treatment of Methicilin-Resistant Staphylococcus aureus infections in adults and children. Clinical Infectious Diseases 2011; 52: 1-38.

65. LO, W.T. and Wang, C.C. Panton-Valentine leukocidin in the pathogenesis of community-associated methicillin-resistant Staphylococcus aureus infection. (2011) Pediatr Neonatol. 52(2), 59-65.

66. LU, P. L. et al. Risk factors and molecular analysis of community methicillin-resistant Staphylococcus aureus carriage. J Clin Microbiol, 43(1), 132-139, 2005.

67. N.GARDELLA; S.Murzicato; S. Di Gregorio; A. Cuirolo; J.Desse; F. Crudo; G.Gutking; M. Mollerach. Prevalence and Characterization of methicilin- resistent Sthaphylococcus aureus among healthy children in city of Argentina. Infection, Genetics and Evolution 11 (2011) 1066-1071

68. OKUMA. K. et al. Dissemination of new methicillinresistant Staphylococcus aureus clones in the community. J Clin Microbiol, 40: 4289 - 4294, 2002.

69. OLIVEIRA, D.C.; Tomasz, A. and de Lencastre, $H$. The evolution of pandemic clones of methicillinresistant Staphylococcus aureus: identification of two ancestral genetic backgrounds and the associated mec elements. (2001) Microb Drug Resist. 7(4), 349-361.

70. OLIVEIRA, G.A.; Faria, J.B.; Levy, C.E. and Mamizuka, E.M. Characterization of the Brazilian endemic clone of methicillin-resistant Staphylococcus aureus (MRSA) from hospitals throughout Brazil. (2001) Braz J Infect Dis. 5(4), 163-170.

71. OTTO, M. A MRSA-terious enemy among us: end of the PVL controversy? (2011) Nat Med. 17(2), 169-170.

72. RAMOS, R.L.; Teixeira, L.A.; Ormonde, L.R.; Siqueira, P.L.; Santos, M.S.; Marangoni, D. and Figueiredo, A.M. Emergence of mupirocin resistance in multiresistant Staphylococcus aureus clinical isolates belonging to Brazilian epidemic clone III::B:A. (1999) J Med Microbiol. 48(3), 303-307.
73. RIZEK, C.F.; Matte, M.H.; Dropa, M.; Mamizuka, E.M.; de Almeida, L.M.; Lincopan, N.; Matte, G.R. and Germano, P.M. Identification of Staphylococcus aureus carrying the mecA gene in ready-to-eat food products sold in Brazil. (2011) Foodborne Pathog Dis. 8(4), 561-563.

74. ROBINSON, D.A. and Enright, M.C. Multilocus sequence typing and the evolution of methicillin-resistant Staphylococcus aureus. (2004) Clin Microbiol Infect. 10(2), 92-97.

75. RODRIGUEZ-NORIEGA, E.; Seas, C.; GuzmanBlanco, M.; Mejia, C.; Alvarez, C.; Bavestrello, L.; Zurita, J.; Labarca, J.; Luna, C.M.; Salles, M.J. and Gotuzzo, E. Evolution of methicillin-resistant Staphylococcus aureus clones in Latin America. (2010) Int J Infect Dis. 14(7), e560-566.

76. ROZENBAUM, R.; Sampaio, M.G.; Batista, G.S.; Garibaldi, A.M.; Terra, G.M.; Souza, M.J.; Vieira, E.N.; Silva-Carvalho, M.C.; Teixeira, L.A. and Figueiredo, A.M. The first report in Brazil of severe infection caused by community-acquired methicillinresistant Staphylococcus aureus (CA-MRSA). (2009) Braz J Med Biol Res. 42(8), 756-760.

77. ROZENBAUM, R.; Silva-Carvalho, M.C.; Souza, R.R.; Melo, M.C.; Gobbi, C.N.; Coelho, L.R.; Ferreira, R.L.; Ferreira-Carvalho, B.T.; Schuenck, A.L.; Neves, F.M.; Silva, L.R. and Figueiredo, A.M. Molecular characterization of methicillin-resistant Staphylococcus aureus disseminated in a home care system. (2006) Infect Control Hosp Epidemiol. 27(10), 1041-1050.

78. SADER, H.S.; Pignatari, A.C.; Hollis, R.J. and Jones, R.N. Evaluation of interhospital spread of methicillin-resistant Staphylococcus aureus in Sao Paulo, Brazil, using pulsed-field gel electrophoresis of chromosomal DNA. (1994) Infect Control Hosp Epidemiol. 15(5), 320-323.

79. SHORE, A.C.; Deasy, E.C.; Slickers, P.; Brennan, G.; O'Connell, B.; Monecke, S.; Ehricht, R. and Coleman, D.C. Detection of Staphylococcal Cassette Chromosome mec Type XI Carrying Highly Divergent mecA, mecI, mecR1, blaZ, and ccr Genes in Human Clinical Isolates of Clonal Complex 130 Methicillin-Resistant Staphylococcus aureus. (2011) Antimicrob Agents Chemother. 55(8), 3765-3773. 
80. SOARES, M.J.; Teixeira, L.A.; Nunes, M.R.; da Silva Carvalho, M.C.; Ferreira-Carvalho, B.T. and Figueiredo, A.M. Analysis of different molecular methods for typing methicillin-resistant Staphylococcus aureus isolates belonging to the Brazilian epidemic clone. (2001) J Med Microbiol. 50(8), 732-742.

81. SOUZA de A. M.; Lencastre de H. Bridges from hospitals to the laboratory: genectic portraits of methicilinresistent Sthaphylococcus aureus clones. FEMS Immunology and Medical Microbiology 40 (2004) 101-111.

82. TENOVER, F.C.; Arbeit, R.D.; Goering, R.V.; Mickelsen, P.A.; Murray, B.E.; Persing, D.H. and Swaminathan, $\mathrm{B}$. Interpreting chromosomal DNA restriction patterns produced by pulsed-field gel electrophoresis: criteria for bacterial strain typing. (1995) J Clin Microbiol. 33(9), 2233-2239.

83. ZHANG, K.; McClure, J.A.; Elsayed, S. and Conly, J.M. Novel staphylococcal cassette chromosome mec type, tentatively designated type VIII, harboring class A mec and type 4 ccr gene complexes in a Canadian epidemic strain of methicillin-resistant Staphylococcus aureus. (2009) Antimicrob Agents Chemother. 53(2), 531-540.

84. CARVALHO, A.M.P. e GIL-PÉREZ, D. Formação de professores de ciências: tendências e inovaçóes. 3. ed. São Paulo: Cortez, 1998.
85. CACHAPUZ, A., Gil-Perez, D., Carvalho, A.M.P., Praia, J. e Vilches, A . A Necessária Renovação do Ensino das Ciências. São Paulo, Editora Cortez, 2005.

86. FOUREZ, G. A. Crise no ensino de ciências? Investigaçôes em Ensino de Ciências, v.8(2),2003.Em http:// www.if.ufrgs.br/ienci/artigos/Artigo ID99/v8 n2 a2003.pdf.

87. GIORDAN, A. e DE VECHI, G. As origens do saber das concepçóes dos aprendentes aos conceitos cientificos. Porto Alegre: Artes Médicas, 1996.

88. MITRE et al., 2008.

\section{AGRADECIMENTOS}

Nós agradecemos a colaboração do Programa de PósGraduação em Patologia da Universidade Federal Fluminense e o Financiamento do Conselho Nacional de Desenvolvimento Científico e Tecnológico - CNPq para os trabalhos desenvolvidos por grupo.

Endereço para Correspondência:

Fábio Aguiar Alves - faalves@vm.uff.br

Polo Universitário de Nova Friburgo - Universidade Federal Fluminense

Rua Doutor Silvio Henrique Braune, 22 - Centro

Nova Friburgo - RJ - CEP: 28625-650

Tel.: (22) 2528-7168 